\title{
Stagnant slab front within the mantle transition zone controls the formation of Cenozoic intracontinental high-Mg andesites in NE Asia
}

Wen-Liang $\mathrm{Xu}^{1^{*}}$, Jia-Hui Chen ${ }^{1}$, Ai-Hua-Weng ${ }^{2}$, Jie Tang ${ }^{1}$, Feng Wang ${ }^{1}$, Chun-Guang Wang ${ }^{1}$, Peng Guo ${ }^{1}$, Yi-Ni Wang ${ }^{1}$, Hao Yang ${ }^{1}$, A.A. Sorokin ${ }^{3}$

${ }^{1}$ College of Earth Sciences, Jilin University, Changchun 130061, China

2 College of Geo-Exploration Science and Technology, Jilin University, Changchun 130061, China

${ }^{3}$ Institute of Geology and Nature Management, Far Eastern Branch of the Russian Academy of Sciences, Blagoveshchensk 675000, Russia 


\section{Supplemental Material}

Analytical methods, supplementary figures and tables

Analytical methods

Supplementary Figure DR1

Supplementary Figure DR2

Supplementary Figure DR3

Text S1 (including Table DR7)

Supplementary Figure DR4

Supplementary Figure DR5

Supplementary Figure DR6

Supplementary References

Supplementary Table DR1

Supplementary Table DR2

Supplementary Table DR3

Supplementary Table DR4

Supplementary Table DR5

Supplementary Table DR6 


\section{Analytical methods}

\section{Mineral electron probe analyses}

The mineral phases were performed for major elements on a four-spectrometer Jeol JXA 8100 electron probe microanalyzer in the Key Laboratory of Submarine Geoscience, State Oceanic Administration, using an accelerating potential of $15 \mathrm{kV}$, a beam current of $20 \mathrm{nA}$, a counting time of $20 \mathrm{~s}$, and a spot size of 5um. Natural mineral standards and ZAF correction scheme were used for calibration. The analytical results of representative minerals are presented in Table DR1.

\section{Whole-rock major and trace element determinations}

For geochemical analysis, whole-rock samples were crushed in an agate mill to $\sim 200$ mesh. X-ray fluorescence (XRF; Rigaku RIX 2100 spectrometer) using fused glass disks and ICP-MS (Agilent 7500a with a shield torch) were used to measure the major and trace elements compositions, respectively, at the State Key Laboratory of Geological Processes and Mineral Resources, China University of Geosciences, after acid digestion of samples in Teflon bombs. Analytical uncertainties are in the range $1-3 \%$. The analytical results for the BHVO-1 (basalt), BCR-2 (basalt), and AGV-1 (andesite) standards indicate that the analytical precision for major elements is better than $5 \%$, and for trace elements, generally better than 10\% (Rudnick et al., 2004). Table DR2 lists the analytical results of major and trace elements for high-Mg andesites in Russian Far East. 


\section{Sr-Nd-Pb-Hf isotope analyses}

Bulk-rock Sr-Nd-Pb-Hf isotope ratios were measured using a Nu Plasma MC-ICP-MS in the Institute of Oceanology, Chinese Academy of Sciences (IOCAS). About $50 \mathrm{mg}$ of rock powder was dissolved with double distilled $\mathrm{HNO}_{3}+\mathrm{HCl}+\mathrm{HF}$ in a high-pressure jacket equipped Teflon beaker at $190^{\circ} \mathrm{C}$ for 15 hours, which was then dried and re-dissolved with $2 \mathrm{ml} 3 \mathrm{~N} \mathrm{HNO}_{3}$ for $2 \mathrm{~h}$. The final sample solution was first loaded onto Sr-spec resin columns to separate $\mathrm{Sr}$ and $\mathrm{Pb}$, with the eluted sample solution collected and then loaded onto AG 50W-X8 resin columns to separate REE. The eluted sample solution from AG 50W-X8 resin columns was collected and then loaded onto Ln-spec resin columns to collect Hf. The separated REE solution was dried and re-dissolved with $0.25 \mathrm{~N} \mathrm{HCl}$ before being loaded onto Ln-spec resin columns to collect $\mathrm{Nd}$. The measured ${ }^{87} \mathrm{Sr} /{ }^{86} \mathrm{Sr},{ }^{143} \mathrm{Nd} /{ }^{144} \mathrm{Nd}$ and ${ }^{176} \mathrm{Hf} /{ }^{177} \mathrm{Hf}$ isotope ratios were normalized for instrumental mass fraction using the exponential law to ${ }^{86} \mathrm{Sr} /{ }^{88} \mathrm{Sr}=0.1194,{ }^{146} \mathrm{Nd} /{ }^{144} \mathrm{Nd}=0.7219$ and ${ }^{179} \mathrm{Hf} /{ }^{177} \mathrm{Hf}=0.7325$, respectively. International standards of NBS-987, JNdi-1 and Alfa Hf were used as bracketing standards every five samples to monitor the instrument drift during the analysis of $\mathrm{Sr}, \mathrm{Nd}$ and $\mathrm{Hf}$ isotopes, respectively. Repeated analysis for NBS-987 gives the average of ${ }^{87} \mathrm{Sr} /{ }^{86} \mathrm{Sr}$. Repeated analysis for JNdi-1 gives the average of ${ }^{143} \mathrm{Nd} /{ }^{144} \mathrm{Nd}$, and repeated analysis for Alfa $\mathrm{Hf}$ gives the average of ${ }^{176} \mathrm{Hf} /{ }^{177} \mathrm{Hf}$. Pb isotope ratios were normalized for instrumental mass fraction relative to NBS/SRM $997^{203} \mathrm{TI} /{ }^{205} \mathrm{TI}=0.41891$. The international 
standard NBS-981 was used to monitor the instrument drift during the analysis of $\mathrm{Pb}$ isotopes. Repeated analysis of NBS-981 gives the average of ${ }^{206} \mathrm{~Pb} /{ }^{204} \mathrm{~Pb},{ }^{207} \mathrm{~Pb} /{ }^{204} \mathrm{~Pb}$ and ${ }^{208} \mathrm{~Pb} /{ }^{204} \mathrm{~Pb}$. Due to machine damage, the test result of $\mathrm{Sr}$ isotope ratio was completed by using TRITON thermoelectric ionization mass spectrometry in Tianjin Geological Survey Center . The main steps of testing Sr sample by TIMS are as follows: take the Nd standard sample of about $100 \mathrm{ng}$, apply it to the center of Re band after degassing, dry it under the electric current of $0.5 \mathrm{~A}$, slowly increase the electric current (about 2.2A) to the Re band in dark red, keep it for $10-20$ s, then decrease the current and make it cool down. The evaporation band and ionization band are installed on the sample plate and sent to the sample room for testing. During the test, the vacuum of the sample chamber is lower than $9^{*} 10^{-8} \mathrm{~Pa}$, and the vacuum of the analysis chamber is lower than $9 * 10^{-9} \mathrm{~Pa}$. Generally, the ionization temperature of $\mathrm{Sr}$ is about $1250 \sim 1350^{\circ} \mathrm{C}$. The ionic flow strength is generally controlled above $5 \mathrm{~V}$. The analytical results of $\mathrm{Sr}-\mathrm{Nd}-\mathrm{Pb}-\mathrm{Hf}$ isotopes for studied samples are listed in Table DR3. 


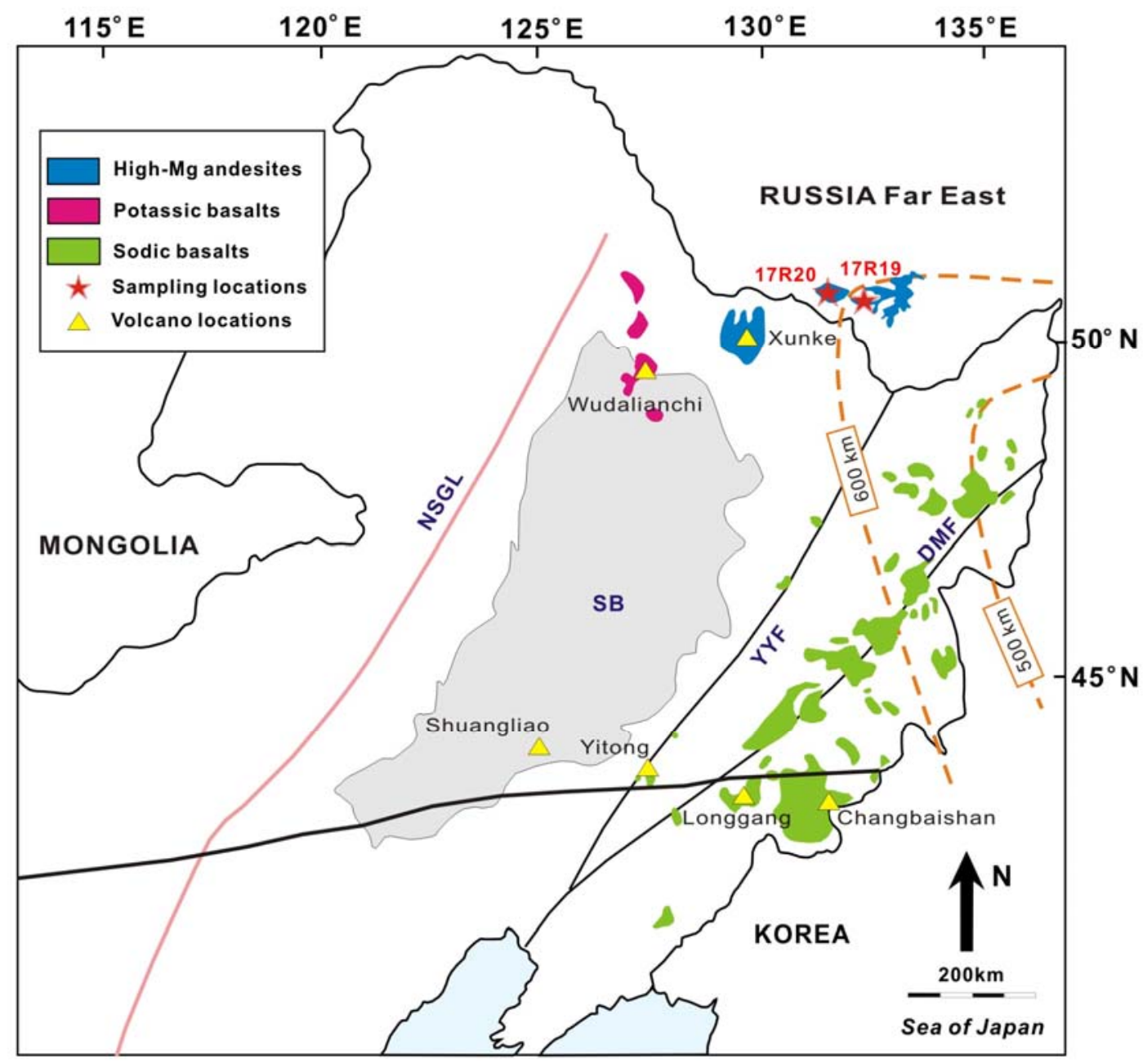

Figure DR1. Distribution map of the Cenozoic volcanic rocks to east of the North-South Gravity Lineament (NSGL). Dashed yellow lines represent the depth of the Wadati-Benioff earthquake zone (Zhao et al., 2009). DMF: Dunhua-Mishan Fault; SB: Songliao Basin; YYF: Yilan-Yitong Fault. 

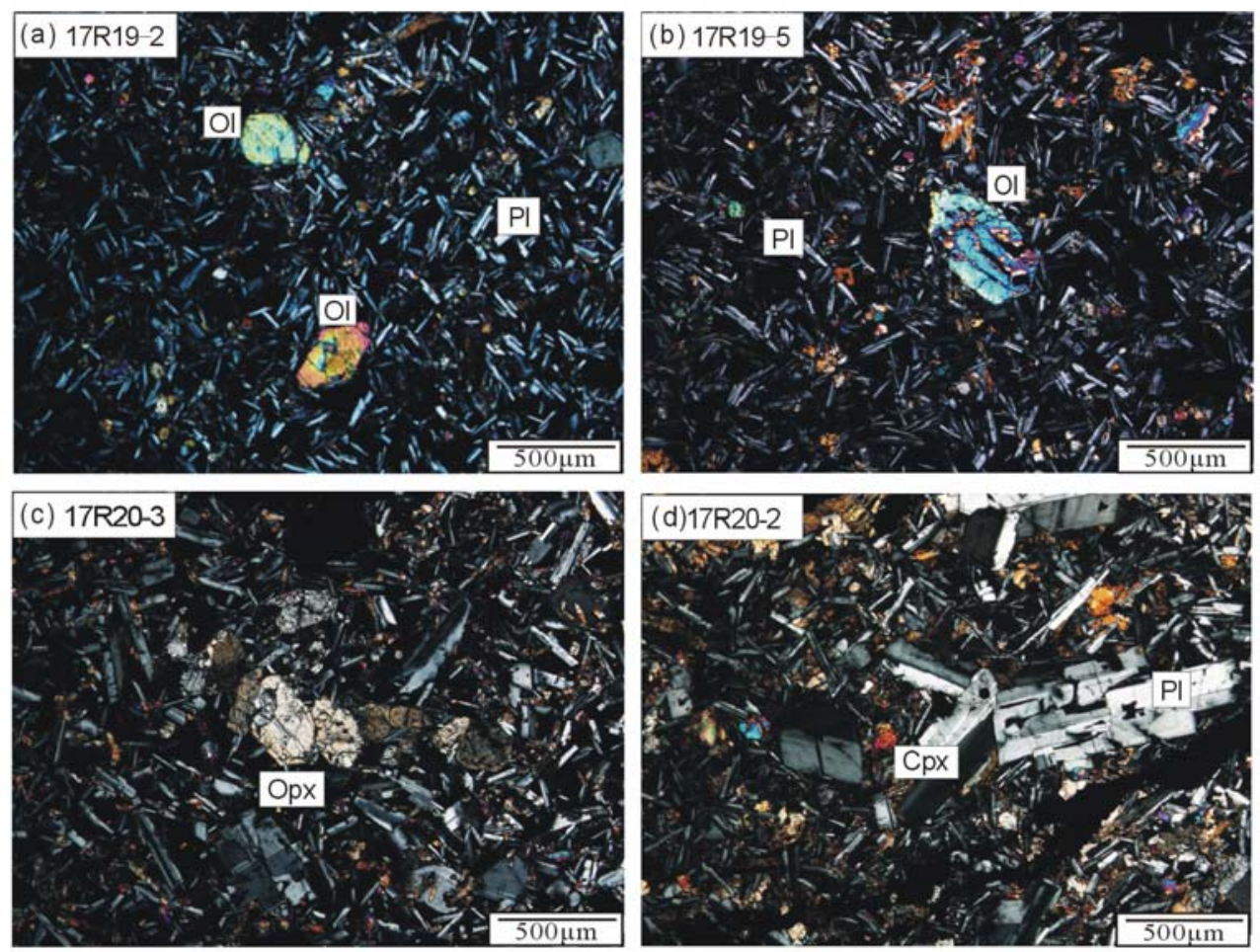

Figure DR2. Representative microphotographs of the Cenozoic intracontinental high-Mg andesites from the Russian Far East. Microphotographs (crossed polarized light) showing the mineral composition, texture and structure of the high-Mg andesites sampled during this study. Abbreviations are as follows: $\mathrm{Cpx}=$ clinopyroxene; Ol = olivine; Opx = orthopyroxene; $\mathrm{PI}=$ plagioclase . 

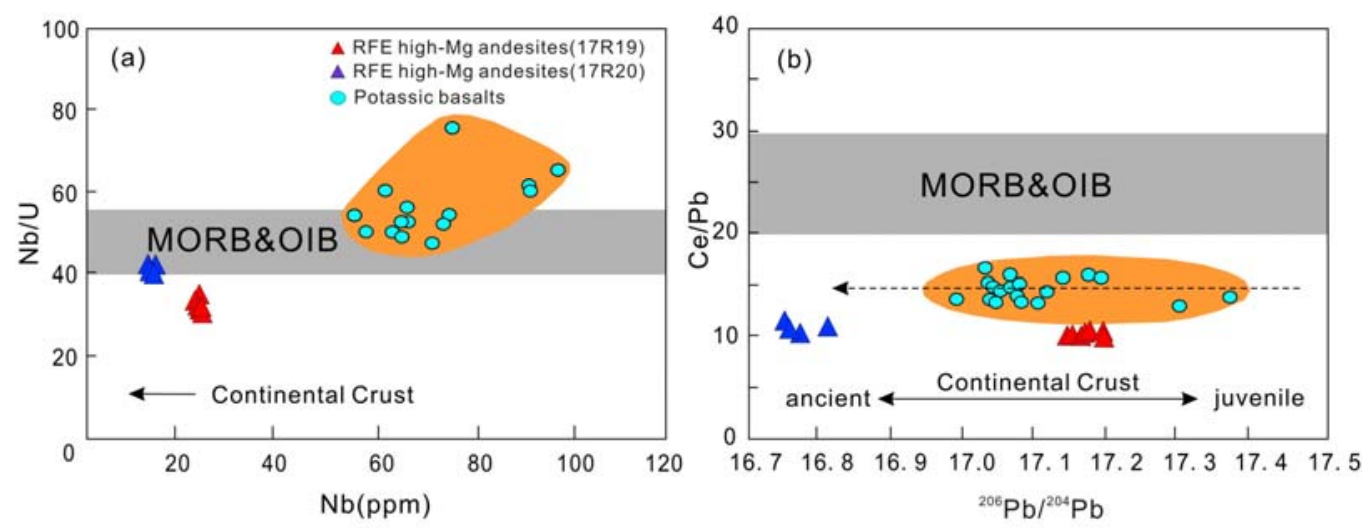

Figure DR3. Plots of $\mathrm{Nb} / \mathrm{U}$ ratios versus $\mathrm{Nb}(\mathrm{a})$ and $\mathrm{Ce} / \mathrm{Pb}$ ratios versus ${ }^{206} \mathrm{~Pb} /{ }^{204} \mathrm{~Pb}(\mathrm{~b})$ for the Cenozoic intracontinental high-Mg andesites from the Russian Far East and MORB, OIB, and continental crust (Date from Hofmann et al., 1986). Data of the Cenozoic potassic basalts in NE China from Basu et al. (1991), Zhang et al. (1991, 1995), and Zou et al. (2003). 


\section{Text S1}

\section{Text S1 Petro-physical constraints of the anomalies beneath NE China}

To estimate water content and its possible derived partial melting in the asthenosphere, we can follow steps bellow:

1. Water content and its uncertainty are estimated for the resistivity and temperature at a certain depth according to (Gardés et al., 2014) (Fig DR4);

2. Constructing local geotherm from Ichiki et al. (2006) (Fig DR5);

3. Water storage capacity at the same depth is estimated according to (Hirschmann et al., 2009) after Fig DR5;

4. If estimated water content is less than the corresponding storage capacity, partial melting cannot be triggered, we will use water to interpret low resistivity anomaly. Otherwise, partial melting can occur and we go to next step;

5. Using the water storage capacity and the partition coefficient in (Dmantle/melt=0.006) (Hirschmann et al., 2009) to estimate water content in molten phase, from which and considering local geothermal temperature, we estimate melt conductivity by (Ni et al., 2011);

6. Using dual-phase mixed formula from (Dulien, 1979) with $0.01 \mathrm{~S} / \mathrm{m}$ as matrix conductivity, the melting fraction could be evaluated.

Thea above steps could be applied to interpret the resistivity of anomalies A, B and C at depth about $325 \mathrm{~km}$. The water content and possible melting fractions are estimated and listed in the Table DR7. 
Table DR7 Summary of the rock-physics parameters of the detected anomalies.

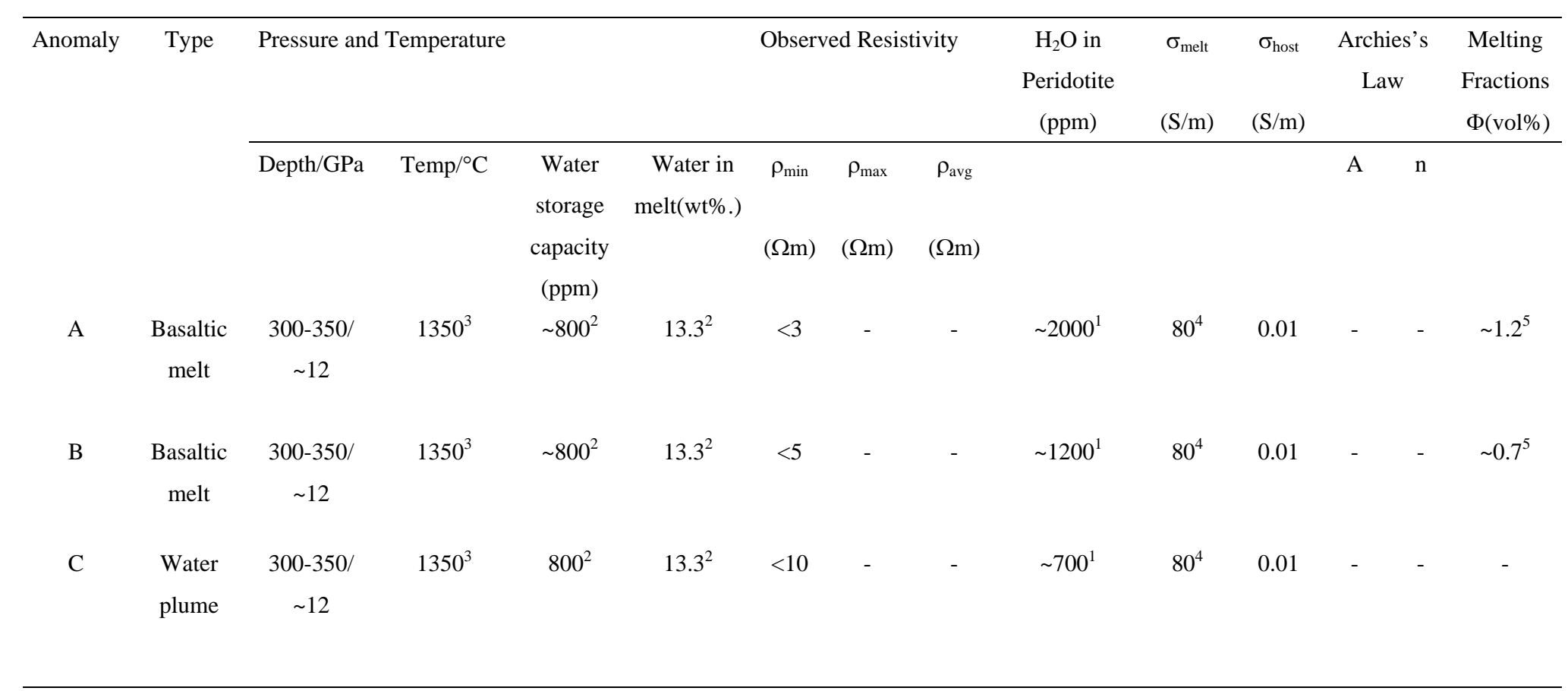

${ }^{1}$ General formula for peridotite conductivity from Ref. (Gardés et al.,2014).

${ }^{2}$ Water content in melt using partition coefficients $D^{\text {mantle/melt }}=0.006$ from Ref. (Hirschmann et al., 2009).

${ }^{3}$ Geothermal temperature from Ref. (Ichiki et al., 2006).

${ }^{4}$ Basaltic melt conductivity from Ref. (Ni et al., 2011).

${ }^{5}$ Lower crust and mantle: dual-phase mixed formula from Ref. (Dulien, 1979). 


\section{Fig.DR4}

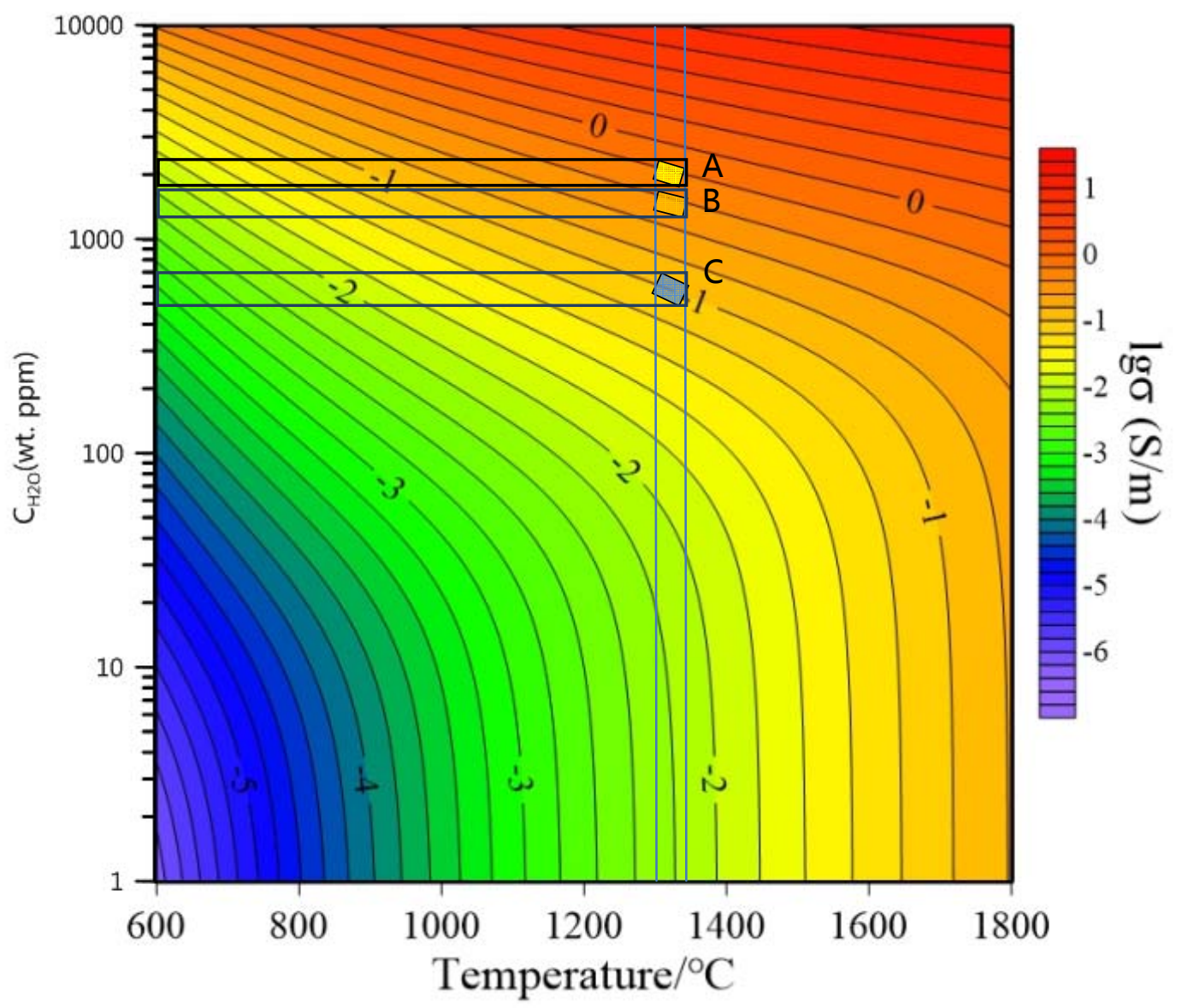

Fig.DR4. Bulk resistivity (in log units) of olivine as a function of temperature and water content (Gardés et al.,2014). Resistivities at the center of anomalies A, B and C are as low as 3, 5, and $10 \Omega \cdot \mathrm{m}$ at $350 \mathrm{~km}$ depth where the geotherm temperature is estimated to be about $1350^{\circ} \mathrm{C}$ (Ichiki et al., 2006). 
Fig.DR5

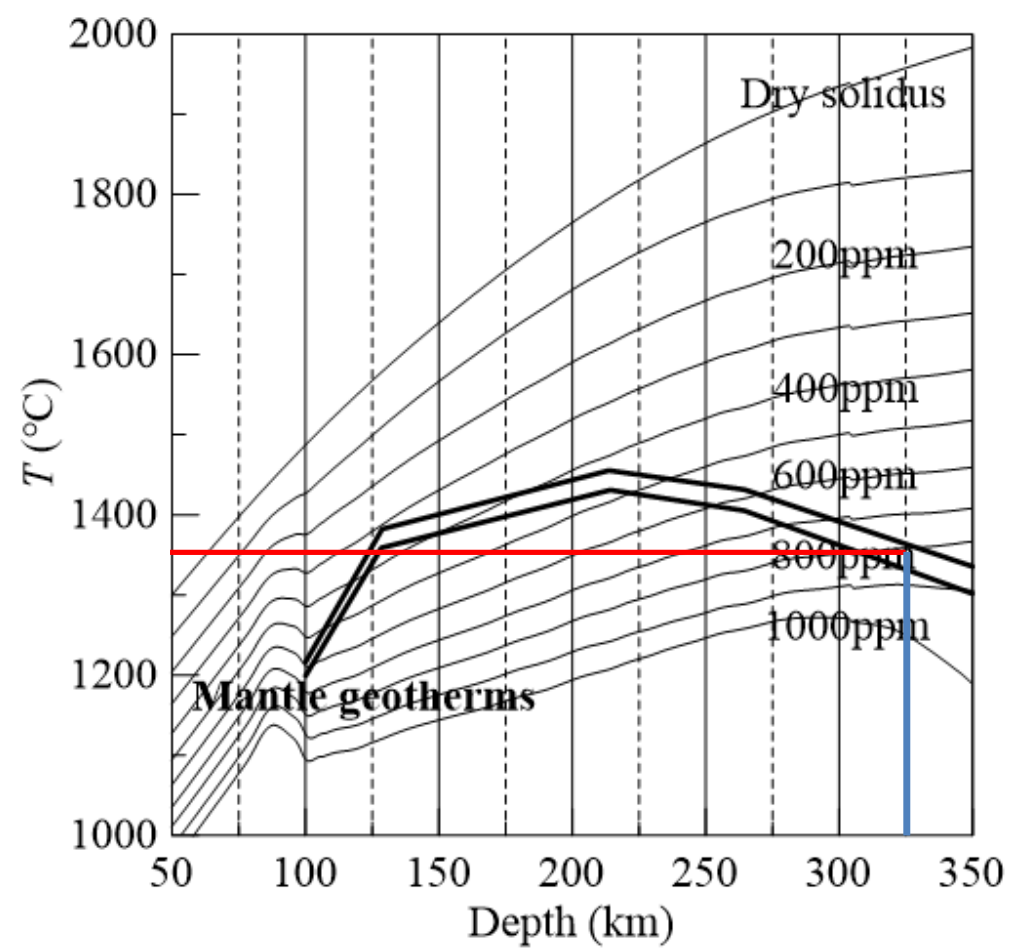

Fig.DR5. Peridotite water storage capacity beneath NE China. Peridotite solidus (thin lines) at different water content (Hirschmann et al,2009). Thick solid lines are the upper and lower bounds of the wave velocity derived geotherm beneath NE China (Ichiki et al., 2006). Water storage capacity in asthenosphere beneath NE China could be read from the cross-points of the geothermal curves with solidus lines at different water content. Water storage capacity is a water content when exceeding this content partial melting will occur, and once partial melting onset, most water in mantle rock will partition into melt, leaving a dry matrix. Water storage capacity increase with pressure, but is also affected by temperature. For example, at the base of asthenosphere where temperature is $1350^{\circ} \mathrm{C}$, the water storage capacity rises to $\sim 800 \mathrm{ppm}$. However, if using normal geothermal, about $1400{ }^{\circ} \mathrm{C}$, it will drop to $\sim 500 \mathrm{ppm}$. 


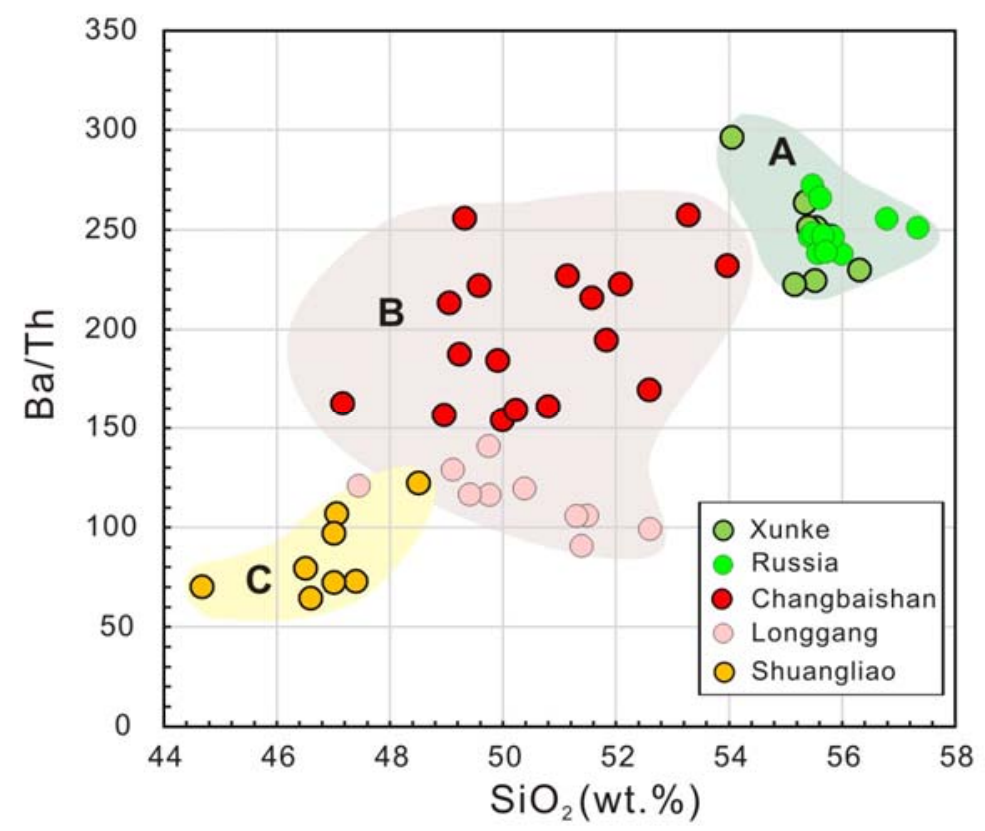

Fig.DR6. Plot of $\mathrm{Ba} / \mathrm{Th}$ versus $\mathrm{SiO}_{2}$ for the Cenozoic volcanic rocks corresponding for three low-resistivity anomalies A, B, C in NE Asia. Generally, $\mathrm{Ba} / \mathrm{Th}$ ratios of volcanic rocks in arcs have positive correlation with water content in magma sources (Chen et al., 2017). The Cenozoic intracontinental HMAs in NE Asia related to anomaly $A$ have the largest $\mathrm{Ba} / \mathrm{Th}$ ratios (238-273) and the highest $\mathrm{SiO}_{2}$ contents (55.4\%-57.3\%), implying their highest water content; the Changbaishan and Longgang sodic basalts related to anomaly $\mathrm{B}$ have moderate $\mathrm{Ba} / \mathrm{Th}$ ratios $(90-266)$ and $\mathrm{SiO}_{2}$ contents (46.2\%-53.9\%), i.e., moderate water content; whereas the Shuangliao sodic basalts related to anomaly $\mathrm{C}$ exhibit the lowest $\mathrm{Ba} / \mathrm{Th}$ ratios (64-122) and $\mathrm{SiO}_{2}$ contents (42.8\%-48.5\%), i.e., the lowest water content. 


\section{SUPPLEMENTARY REFERENCES}

Basu, A.R., Wang, J.W., Huang, W.K., Xie, G.H., Tatsumoto, M., 1991, Major element, $\mathrm{REE}$, and $\mathrm{Pb}, \mathrm{Nd}$ and $\mathrm{Sr}$ isotopic geochemistry of Cenozoic volcanic rocks of eastern China: implications for their origin from suboceanic-type mantle reservoirs: Earth and Planetary Science Letters, v. 105, p.149-169, https://doi: 10.1016/0012-821X(91)90127-4.

Chen, H., Xia, Q.K., Ingrin, J., Deloule, E., Bi, Y., 2017, Heterogeneous source components of intraplate basalts from NE China induced by the ongoing Pacific slab subduction: Earth and Planetary Science Letters, v.459, p. 208-220, http://dx.doi.org/10.1016/j.epsl.2016.11.030

Dulien, F.A.L., 1979, Porous Media: Fluid transport and pore structure. Academic Press, New York, pp. 396.

Hirschmann, M.M., Tenner, T., Aubaud, C., Withers, A.C., Pepi, P., 2009, Dehydration melting of nominally anhydrous mantle: The primacy of partitioning. Physics of the Earth and Planetary Interiors, v. 176, p. 54-68, https://doi:10.1016/j.pepi.2009.04.001.

Hofmann, A.-W., Jochum, K.-P., Seufert, M., White, W.-M., 1986, \#b and Pb in oceanic basalts: new constraints on mantle evolution: Earth and Planetary Science Letters, v. 79: p. 33-45, https://doi.10.1016/0012- 821x(86)90038 -5 .

Ma, Q., Xu, Y. G., Zheng, J. P., Griffin, W. L., Hong, L. B., \& Ma, L., 2016, Coexisting Early Cretaceous high - Mg andesites and adakitic rocks in the North China Craton: The role of water in intraplate magmatism and cratonic destruction: Journal of Petrology, v. 57(7), p. 1279-1308, https://doi.org/10.1093/petrology/ egw040.

Ni, H. W., Keppler, H. \& Behrens, H., 2011, Electrical conductivity of hydrous basaltic melts: implications for partial melting in the upper mantle: Contributions to Mineralogy and Petrology, v.162, p. 637-650, 
https://doi:10.1007/s00410-011- 0617-4.

Rudnick, R. L., Gao, S., Ling, W. L., Liu, Y. S., McDonough, W. F., 2004, Petrology and geochemistry of spinel peridotite xenoliths from Hannuoba and Qixia, North China Craton: Lithos, v. 77, p. 609-637, https://doi: 10.1016/j.lithos.2004.03.033.

Zhang, M., Menzies, M.A., Suddaby, P., Thirlwall, M.F., 1991, EM1 signature from within the post-Archaean subcontinental lithospheric mantle: isotopic evidence from the potassic volcanic rocks in NE China: Geochemical Journal, v. 25, p. 387-398, https://doi:10.2343/geochemj.25.387.

Zhang, M., Suddaby, P., Thompson, R.N., Thirlwall, M.F.,Menzies,M.A., 1995, Potassic volcanic rocks in NE China: geochemical constraints onmantle source and magma genesis: Journal of Petrology, v. 36, p.1275-1303, https://doi.org/ 10.1093/petrology/36.5.1275.

Zou, H., Fan, Q. \& Yao, Y., 2008, U-Th systematic of dispersed young volcanoes in NE China: asthenosphere upwelling caused by pilling up and upward thickening of stagnant Pacific slab: Chemical Geology, v. 255, p.134-142, https://doi:10.1016/j.chemgeo.2008.06.022.

Zou, H., Reid, M.R., Liu, Y., Yao, Y., Xu, X., Fan, Q., 2003, Constraints on the origin of historic potassic basalts fromnortheast China by UITh disequilibrium data: Chemical Geology, v. 200, p.189-201, https://doi. 10.1016/S0009-2541(03) 00188-8.

Table DR1 Mineral chemical data (wt.\%) for the Cenozoic intracontinental high-Mg andesites from the Russian Far East

Table DR2 Major (wt\%) and trace elements (ppm) data for the Cenozoic intracontinental high-Mg andesites from the Russian Far East 
Table DR3 Sr-Nd-Hf-Pb isotopic data of the Cenozoic intracontinental high-Mg andesites from the Russian Far East

Table DR4 Geochemical characteristics of some high-Mg andesites (wt.\%) from subduction zones

Table DR5 Compared $\mathrm{Sr}-\mathrm{Nd}-\mathrm{Pb}$ isotopic data from intraplate basalts in NE China and andesites in arcs

Table DR6 Present-day compositions of the end-member components 
Table DR1 Mineral chemical data (wt.\%) for the Cenozoic intracontinental high-Mg andesites from Russian Far East

\begin{tabular}{|c|c|c|c|c|c|c|c|c|c|c|c|c|c|c|c|c|c|c|c|}
\hline \multirow{2}{*}{ Sample } & \multicolumn{5}{|c|}{ 17R19-2 } & \multicolumn{4}{|c|}{ 17R19-5 } & \multicolumn{6}{|c|}{ 17R20-2 } & \multicolumn{4}{|c|}{ 17R20-3 } \\
\hline & $19-2-1$ & $19-2-2$ & $19-2-3$ & $19-2-4$ & $19-2-5$ & 19-5-1 & $19-5-2$ & $19-5-3$ & $19-5-4$ & $20-2-1$ & $20-2-2$ & $20-2-4$ & $20-2-5$ & $20-2-6$ & $20-2-7$ & $20-3-1$ & $20-3-2$ & $20-3-3$ & $20-3-4$ \\
\hline Mineral & $\mathrm{Ol}$ & $\mathrm{Ol}$ & $\mathrm{Ol}$ & $\mathrm{Pl}$ & $\mathrm{Ol}$ & $\mathrm{Ol}$ & $\mathrm{Ol}$ & $\mathrm{Pl}$ & $\mathrm{Ol}$ & $\mathrm{Ol}$ & $\mathrm{Ol}$ & $\mathrm{Pl}(\mathrm{r})$ & $\mathrm{Pl}(\mathrm{c})$ & Cpx (c) & Cpx(c) & Opx (c) & $\mathrm{Pl}(\mathrm{C})$ & $\mathrm{Pl}(\mathrm{r})$ & Opx (r) \\
\hline $\mathrm{SiO}_{2}$ & 39.50 & 38.89 & 39.02 & 55.91 & 38.95 & 39.16 & 39.41 & 56.91 & 39.01 & 40.03 & 38.63 & 56.32 & 55.68 & 52.25 & 52.14 & 54.32 & 54.66 & 57.09 & 54.33 \\
\hline $\mathrm{TiO}_{2}$ & 0.03 & - & - & 0.09 & - & 0.02 & - & 0.11 & - & - & - & 0.06 & 0.03 & 0.82 & 0.82 & 0.28 & 0.06 & 0.04 & 0.33 \\
\hline $\mathrm{Al}_{2} \mathrm{O}_{3}$ & 0.08 & 0.04 & 0.02 & 26.65 & 0.03 & 0.06 & 0.03 & 26.01 & - & 0.04 & 0.02 & 26.84 & 26.96 & 1.75 & 2.09 & 2.40 & 27.48 & 26.14 & 1.32 \\
\hline $\mathrm{Cr}_{2} \mathrm{O}_{3}$ & 0.03 & 0.02 & 0.05 & 0.02 & 0.04 & 0.06 & 0.02 & - & 0.01 & 0.01 & 0.02 & 0.01 & 0.04 & 0.11 & 0.29 & 0.35 & - & 0.02 & 0.45 \\
\hline $\mathrm{FeO}$ & 17.87 & 20.34 & 16.65 & 0.75 & 19.91 & 17.30 & 17.99 & 0.57 & 19.87 & 14.94 & 23.20 & 0.33 & 0.53 & 12.26 & 10.14 & 12.54 & 0.72 & 0.40 & 14.40 \\
\hline $\mathrm{MnO}$ & 0.27 & 0.28 & 0.22 & 0.03 & 0.27 & 0.23 & 0.28 & - & 0.24 & 0.15 & 0.31 & - & 0.01 & 0.32 & 0.27 & 0.26 & - & 0.01 & 0.28 \\
\hline $\mathrm{MgO}$ & 42.68 & 41.22 & 43.40 & 0.15 & 41.16 & 42.81 & 42.49 & 0.13 & 41.04 & 44.78 & 38.20 & 0.11 & 0.13 & 17.44 & 17.23 & 27.84 & 0.17 & 0.11 & 26.30 \\
\hline $\mathrm{CaO}$ & 0.17 & 0.19 & 0.18 & 10.25 & 0.18 & 0.18 & 0.21 & 9.67 & 0.18 & 0.06 & 0.21 & 10.27 & 10.55 & 14.24 & 16.38 & 1.77 & 11.50 & 9.96 & 2.06 \\
\hline $\mathrm{Na} 2 \mathrm{O}$ & 0.04 & 0.09 & - & 5.14 & 0.02 & 0.01 & 0.04 & 5.63 & 0.02 & 0.03 & 0.03 & 5.36 & 5.31 & 0.31 & 0.39 & 0.16 & 4.92 & 5.54 & 0.10 \\
\hline $\mathrm{K}_{2} \mathrm{O}$ & 0.02 & 0.01 & - & 0.53 & 0.03 & - & 0.05 & 0.59 & 0.01 & 0.02 & 0.01 & 0.38 & 0.35 & 0.03 & 0.02 & 0.08 & 0.31 & 0.40 & 0.01 \\
\hline $\mathrm{NiO}$ & 0.29 & 0.22 & 0.23 & 0.04 & 0.25 & 0.22 & 0.25 & - & 0.27 & 0.35 & 0.29 & 0.03 & 0.04 & 0.06 & 0.00 & 0.14 & 0.01 & 0.03 & 0.01 \\
\hline $\mathrm{P}_{2} \mathrm{O}_{5}$ & 0.09 & 0.04 & 0.15 & - & 0.06 & 0.04 & 0.09 & 0.02 & 0.05 & 0.03 & 0.01 & 0.02 & 0.02 & 0.02 & 0.02 & 0.03 & 0.03 & 0.02 & 0.00 \\
\hline Total & 101.07 & 101.32 & 99.92 & 99.54 & 100.88 & 100.08 & 100.85 & 99.62 & 100.69 & 100.43 & 100.93 & 99.73 & 99.64 & 99.60 & 99.79 & 100.16 & 99.86 & 99.73 & 99.59 \\
\hline Mg\# & 81.0 & 78.3 & 82.3 & 26.0 & 78.7 & 81.5 & 80.8 & 28.7 & 78.6 & 84.2 & 74.6 & 37.6 & 30.5 & 71.7 & 75.2 & 79.8 & 30.3 & 33.2 & 76.5 \\
\hline An & - & - & - & 50.80 & - & - & - & 47.05 & - & - & - & 50.29 & 51.30 & - & - & - & 55.34 & 48.69 & - \\
\hline En & - & - & - & - & - & - & - & - & - & - & - & - & - & 49.65 & 48.75 & 76.29 & - & - & 72.74 \\
\hline Fs & - & - & - & - & - & - & - & - & - & - & - & - & - & 20.08 & 16.50 & 19.67 & - & - & 22.81 \\
\hline Wo & - & - & - & - & - & - & - & - & - & - & - & - & - & 29.12 & 33.31 & 3.48 & - & - & 4.09 \\
\hline Fo & 80.8 & 78.1 & 82.1 & - & 78.4 & 81.3 & 80.6 & - & 78.4 & 84.1 & 74.3 & - & - & - & - & - & - & - & - \\
\hline
\end{tabular}

Note: $\mathrm{Mg} \#=100 *(\mathrm{MgO} / 40.31) /\left(\mathrm{MgO} / 40.31+\mathrm{FeO}^{\mathrm{T}} / 71.85\right)$. C-core; r-rim. 
Table DR2 Major (wt\%) and trace elements (ppm) data for the Cenozoic high-Mg andesites in Russian Far East

\begin{tabular}{|c|c|c|c|c|c|c|c|c|c|c|c|c|}
\hline Sample & 17R19-1 & 17R19-2 & 17R19-3 & 17R19-4 & 17R19-5 & 17R19-6 & 17R19-7 & 17R19-8 & 17R20-1 & 17R20-2 & 17R20-3 & 17R20-4 \\
\hline $\mathrm{SiO}_{2}$ & 55.76 & 55.99 & 55.42 & 55.48 & 55.57 & 55.84 & 55.66 & 55.71 & 55.47 & 55.61 & 56.79 & 57.34 \\
\hline $\mathrm{TiO}_{2}$ & 1.69 & 1.69 & 1.64 & 1.65 & 1.66 & 1.68 & 1.67 & 1.67 & 1.48 & 1.50 & 1.55 & 1.57 \\
\hline $\mathrm{Al}_{2} \mathrm{O}_{3}$ & 14.97 & 15.01 & 14.86 & 14.90 & 14.87 & 14.98 & 15.01 & 14.93 & 14.64 & 14.61 & 14.93 & 15.03 \\
\hline $\mathrm{Fe}_{2} \mathrm{O}_{3}$ & 8.73 & 8.63 & 8.66 & 8.75 & 8.89 & 8.61 & 8.60 & 8.85 & 8.88 & 8.88 & 8.67 & 8.58 \\
\hline $\mathrm{MnO}$ & 0.11 & 0.10 & 0.09 & 0.09 & 0.11 & 0.11 & 0.11 & 0.11 & 0.11 & 0.11 & 0.11 & 0.11 \\
\hline $\mathrm{MgO}$ & 5.15 & 4.92 & 4.97 & 5.06 & 5.24 & 5.12 & 5.13 & 5.29 & 5.78 & 5.60 & 5.27 & 5.17 \\
\hline $\mathrm{CaO}$ & 6.03 & 6.06 & 5.82 & 5.83 & 6.00 & 5.99 & 6.02 & 6.01 & 6.60 & 6.62 & 6.51 & 6.56 \\
\hline $\mathrm{Na}_{2} \mathrm{O}$ & 3.59 & 3.70 & 3.86 & 3.87 & 3.60 & 3.54 & 3.50 & 3.61 & 3.59 & 3.55 & 3.50 & 3.59 \\
\hline $\mathrm{K}_{2} \mathrm{O}$ & 2.73 & 2.61 & 2.38 & 2.39 & 2.66 & 2.91 & 2.90 & 2.75 & 1.57 & 1.63 & 1.94 & 1.93 \\
\hline $\mathrm{P}_{2} \mathrm{O}_{5}$ & 0.44 & 0.44 & 0.42 & 0.42 & 0.43 & 0.43 & 0.43 & 0.43 & 0.28 & 0.29 & 0.29 & 0.29 \\
\hline LOI & 0.33 & 0.57 & 0.90 & 0.77 & 0.43 & 0.25 & 0.36 & 0.26 & 0.93 & 0.82 & 0.03 & 0.01 \\
\hline Total & 99.51 & 99.73 & 99.01 & 99.20 & 99.46 & 99.46 & 99.39 & 99.63 & 99.32 & 99.23 & 99.59 & 100.18 \\
\hline Mg\# & 53.9 & 53.1 & 53.2 & 53.4 & 53.9 & 54.1 & 54.2 & 54.2 & 56.3 & 55.5 & 54.6 & 54.4 \\
\hline $\mathrm{Na}_{2} \mathrm{O}+\mathrm{K}_{2} \mathrm{O}$ & 6.32 & 6.31 & 6.24 & 6.27 & 6.26 & 6.45 & 6.41 & 6.36 & 5.15 & 5.18 & 5.44 & 5.52 \\
\hline $\mathrm{Li}$ & 8.06 & 8.38 & 8.18 & 8.59 & 8.26 & 7.83 & 7.99 & 8.54 & 7.04 & 6.97 & 7.18 & 6.96 \\
\hline $\mathrm{Be}$ & 1.39 & 1.38 & 1.38 & 1.46 & 1.48 & 1.47 & 1.52 & 1.44 & 1.30 & 1.32 & 1.33 & 1.43 \\
\hline Sc & 11.1 & 11.0 & 10.9 & 11.0 & 10.9 & 10.9 & 11.3 & 11.3 & 12.1 & 12.2 & 12.6 & 12.6 \\
\hline V & 125 & 126 & 124 & 127 & 125 & 125 & 127 & 127 & 136 & 138 & 139 & 141 \\
\hline $\mathrm{Cr}$ & 160 & 160 & 154 & 159 & 162 & 164 & 164 & 167 & 212 & 197 & 199 & 201 \\
\hline Co & 31.0 & 29.2 & 28.8 & 30.7 & 30.9 & 31.4 & 31.0 & 31.3 & 32.8 & 31.9 & 32.2 & 32.0 \\
\hline $\mathrm{Ni}$ & 96.1 & 92.0 & 89.0 & 95.5 & 95.1 & 98.1 & 97.2 & 97.4 & 161 & 153 & 146 & 143 \\
\hline $\mathrm{Cu}$ & 26.5 & 26.3 & 25.8 & 27.5 & 26.9 & 26.9 & 27.2 & 27.1 & 62.2 & 62.6 & 59.3 & 65.8 \\
\hline $\mathrm{Zn}$ & 102 & 105 & 103 & 103 & 107 & 108 & 108 & 107 & 105 & 105 & 110 & 112 \\
\hline $\mathrm{Ga}$ & 21.9 & 22.3 & 22.1 & 22.2 & 22.0 & 22.6 & 22.8 & 22.8 & 21.8 & 21.4 & 22.3 & 22.6 \\
\hline $\mathrm{Rb}$ & 57.4 & 57.3 & 55.5 & 55.7 & 55.6 & 60.4 & 60.5 & 58.4 & 31.8 & 34.9 & 38.3 & 39.3 \\
\hline $\mathrm{Sr}$ & 733 & 744 & 695 & 711 & 735 & 729 & 748 & 750 & 498 & 496 & 485 & 488 \\
\hline $\mathrm{Y}$ & 17.9 & 18.3 & 17.6 & 17.8 & 18.1 & 18.2 & 18.3 & 18.3 & 15.9 & 16.1 & 17.1 & 17.3 \\
\hline $\mathrm{Zr}$ & 158 & 160 & 152 & 157 & 158 & 158 & 161 & 160 & 138 & 141 & 154 & 158 \\
\hline $\mathrm{Nb}$ & 25.4 & 25.8 & 24.8 & 25.6 & 25.9 & 25.4 & 25.8 & 25.9 & 14.4 & 14.9 & 14.9 & 15.0 \\
\hline Mo & 1.02 & 1.04 & 0.83 & 0.89 & 1.00 & 1.10 & 1.10 & 1.27 & 0.78 & 0.83 & 0.92 & 0.88 \\
\hline Sn & 2.08 & 2.13 & 2.20 & 2.19 & 2.07 & 2.08 & 2.05 & 2.17 & 1.77 & 1.86 & 2.00 & 2.07 \\
\hline Cs & 0.73 & 0.75 & 0.58 & 0.57 & 0.74 & 0.84 & 0.83 & 0.78 & 0.23 & 0.26 & 0.42 & 0.44 \\
\hline $\mathrm{Ba}$ & 759 & 772 & 745 & 767 & 771 & 779 & 790 & 778 & 472 & 486 & 468 & 485 \\
\hline $\mathrm{La}$ & 27.2 & 27.7 & 26.3 & 27.2 & 27.1 & 27.2 & 27.8 & 28.0 & 21.3 & 21.7 & 22.3 & 22.8 \\
\hline $\mathrm{Ce}$ & 56.1 & 57.6 & 54.5 & 56.3 & 56.8 & 56.8 & 58.1 & 57.8 & 42.6 & 43.3 & 44.9 & 45.9 \\
\hline $\operatorname{Pr}$ & 6.91 & 7.07 & 6.57 & 6.83 & 6.88 & 6.90 & 6.88 & 6.86 & 5.03 & 5.16 & 5.42 & 5.53 \\
\hline $\mathrm{Nd}$ & 29.6 & 29.8 & 28.4 & 29.2 & 29.7 & 29.4 & 30.1 & 30.3 & 21.7 & 22.0 & 23.3 & 23.5 \\
\hline $\mathrm{Sm}$ & 6.44 & 6.49 & 6.38 & 6.68 & 6.43 & 6.57 & 6.67 & 6.49 & 4.83 & 4.97 & 5.39 & 5.43 \\
\hline $\mathrm{Eu}$ & 1.87 & 1.86 & 1.85 & 1.95 & 1.88 & 1.93 & 1.98 & 1.90 & 1.54 & 1.56 & 1.58 & 1.65 \\
\hline $\mathrm{Gd}$ & 5.39 & 5.42 & 5.31 & 5.36 & 5.40 & 5.59 & 5.54 & 5.46 & 4.26 & 4.36 & 4.70 & 4.81 \\
\hline $\mathrm{Tb}$ & 0.75 & 0.76 & 0.73 & 0.77 & 0.75 & 0.74 & 0.73 & 0.77 & 0.62 & 0.64 & 0.66 & 0.70 \\
\hline Dy & 3.89 & 3.87 & 3.87 & 3.99 & 3.94 & 4.17 & 4.03 & 4.03 & 3.27 & 3.49 & 3.67 & 3.58 \\
\hline Но & 0.66 & 0.67 & 0.63 & 0.66 & 0.66 & 0.64 & 0.67 & 0.67 & 0.56 & 0.57 & 0.63 & 0.65 \\
\hline $\mathrm{Er}$ & 1.55 & 1.60 & 1.61 & 1.59 & 1.68 & 1.66 & 1.74 & 1.64 & 1.44 & 1.42 & 1.62 & 1.60 \\
\hline $\mathrm{Tm}$ & 0.22 & 0.23 & 0.20 & 0.23 & 0.23 & 0.22 & 0.23 & 0.24 & 0.22 & 0.21 & 0.23 & 0.23 \\
\hline $\mathrm{Yb}$ & 1.18 & 1.21 & 1.09 & 1.21 & 1.16 & 1.15 & 1.23 & 1.19 & 1.06 & 1.05 & 1.22 & 1.27 \\
\hline $\mathrm{Lu}$ & 0.17 & 0.18 & 0.18 & 0.19 & 0.19 & 0.19 & 0.20 & 0.19 & 0.18 & 0.18 & 0.20 & 0.20 \\
\hline Hf & 3.82 & 3.83 & 3.76 & 3.92 & 3.94 & 3.95 & 3.92 & 3.99 & 3.48 & 3.54 & 3.84 & 4.04 \\
\hline Тa & 1.29 & 1.30 & 1.28 & 1.33 & 1.39 & 1.38 & 1.38 & 1.39 & 0.77 & 0.77 & 0.79 & 0.78 \\
\hline W & 0.61 & 0.68 & 0.60 & 0.65 & 0.66 & 0.65 & 0.64 & 0.65 & 0.54 & 0.39 & 0.43 & 0.48 \\
\hline $\mathrm{Tl}$ & 0.18 & 0.16 & 0.16 & 0.16 & 0.17 & 0.18 & 0.16 & 0.19 & 0.095 & 0.10 & 0.14 & 0.13 \\
\hline $\mathrm{Pb}$ & 5.49 & 5.51 & 5.34 & 5.52 & 5.54 & 5.62 & 5.63 & 5.79 & 3.93 & 3.73 & 4.25 & 4.50 \\
\hline Th & 3.16 & 3.24 & 3.02 & 3.09 & 3.24 & 3.16 & 3.20 & 3.26 & 1.73 & 1.83 & 1.83 & 1.93 \\
\hline $\mathrm{U}$ & 0.78 & 0.78 & 0.73 & 0.72 & 0.79 & 0.77 & 0.79 & 0.79 & 0.36 & 0.38 & 0.36 & 0.37 \\
\hline $\mathrm{Nb} / \mathrm{U}$ & 32.45 & 32.96 & 33.98 & 35.51 & 32.70 & 32.81 & 32.65 & 32.73 & 39.60 & 39.47 & 40.85 & 40.55 \\
\hline $\mathrm{Ce} / \mathrm{Pb}$ & 10.22 & 10.44 & 10.19 & 10.19 & 10.25 & 10.11 & 10.33 & 9.99 & 10.84 & 11.62 & 10.56 & 10.21 \\
\hline$(\mathrm{La} / \mathrm{Yb})_{\mathrm{N}}$ & 15.60 & 15.38 & 16.31 & 15.22 & 15.67 & 15.88 & 15.22 & 15.82 & 13.59 & 13.87 & 12.29 & 12.13 \\
\hline$(\mathrm{La} / \mathrm{Sm})_{\mathrm{N}}$ & 2.66 & 2.68 & 2.60 & 2.56 & 2.65 & 2.60 & 2.62 & 2.71 & 2.77 & 2.74 & 2.60 & 2.64 \\
\hline$\sum \mathrm{REE}$ & 141.96 & 144.44 & 137.61 & 142.19 & 142.78 & 143.15 & 145.92 & 145.57 & 108.65 & 110.58 & 115.71 & 117.80 \\
\hline
\end{tabular}

Note: LOI: Loss on ignition; $\sum \mathrm{REE}=\mathrm{La}+\mathrm{Ce}+\mathrm{Pr}+\mathrm{Nd}+\mathrm{Sm}+\mathrm{Eu}+\mathrm{Gd}+\mathrm{Tb}+\mathrm{Dy}+\mathrm{Ho}+\mathrm{Er}+\mathrm{Tm}+\mathrm{Yb}+\mathrm{Lu} ;(\mathrm{La} / \mathrm{Sm})_{\mathrm{N}}=(\mathrm{La} / 0.310) /(\mathrm{Sm} / 0.195)$;

$(\mathrm{La} / \mathrm{Yb})_{\mathrm{N}}=(\mathrm{La} / 0.310) /(\mathrm{Yb} / 0.209) ; \mathrm{Mg} \#=100 *(\mathrm{MgO} / 40.31) /(\mathrm{MgO} / 40.31+\mathrm{FeOT} / 71.85)$. 
Table DR3 Sr-Nd-Hf-Pb isotopes data of high-Mg andesites from Russian Far East.

\begin{tabular}{|c|c|c|c|c|c|c|c|c|c|c|c|c|}
\hline Sample & 17R19-1 & 17R19-2 & 17R19-3 & 17R19-4 & 17R19-5 & 17R19-6 & 17R19-7 & 17R19-8 & $17 R 20-1$ & 17R20-2 & $17 R 20-3$ & 17R20-4 \\
\hline${ }^{87} \mathrm{Sr} /{ }^{86} \mathrm{Sr}$ & 0.705620 & 0.705629 & 0.705670 & 0.705690 & 0.705636 & 0.705632 & 0.705642 & 0.705701 & 0.705862 & 0.705834 & 0.705893 & 0.705890 \\
\hline 1SE & 0.000008 & 0.000007 & 0.000006 & 0.000009 & 0.000008 & 0.000007 & 0.000007 & 0.000010 & 0.000006 & 0.000009 & 0.000009 & 0.000006 \\
\hline${ }^{143} \mathrm{Nd} /{ }^{144} \mathrm{Nd}$ & 0.512432 & 0.512428 & 0.512386 & 0.512411 & 0.512429 & 0.512416 & 0.512419 & 0.512417 & 0.512449 & 0.512463 & 0.512462 & 0.512447 \\
\hline 1SE & 0.000003 & 0.000003 & 0.000002 & 0.000003 & 0.000003 & 0.000003 & 0.000003 & 0.000003 & 0.000002 & 0.000002 & 0.000002 & 0.000003 \\
\hline$\varepsilon_{\mathrm{Nd}}$ & -4.0 & -4.1 & -4.9 & -4.4 & -4.1 & -4.3 & -4.3 & -4.3 & -3.7 & -3.4 & -3.4 & -3.7 \\
\hline${ }^{176} \mathrm{Hf} /{ }^{177} \mathrm{Hf}$ & 0.282640 & 0.282645 & 0.282654 & 0.282646 & 0.282656 & 0.282645 & 0.282652 & 0.282647 & 0.282698 & 0.282686 & 0.282683 & 0.282669 \\
\hline 1SE & 0.000002 & 0.000006 & 0.000002 & 0.000004 & 0.000003 & 0.000002 & 0.000003 & 0.000002 & 0.000003 & 0.000002 & 0.000002 & 0.000004 \\
\hline$\varepsilon_{\mathrm{Hf}}$ & -4.7 & -4.5 & -4.2 & -4.5 & -4.1 & -4.5 & -4.2 & -4.4 & -2.6 & -3.1 & -3.1 & -3.7 \\
\hline${ }^{206} \mathrm{~Pb} /{ }^{204} \mathrm{~Pb}$ & 17.1677 & 17.1846 & 17.1561 & 17.1530 & 17.1870 & 17.1637 & 17.1714 & 17.1866 & 16.8152 & 16.7572 & 16.7634 & 16.7692 \\
\hline 1SE & 0.0002 & 0.0001 & 0.0002 & 0.0001 & 0.0001 & 0.0002 & 0.0002 & 0.0001 & 0.0002 & 0.0002 & 0.0002 & 0.0001 \\
\hline${ }^{207} \mathrm{~Pb} /{ }^{204} \mathrm{~Pb}$ & 15.4432 & 15.4463 & 15.4436 & 15.4443 & 15.4482 & 15.4462 & 15.4463 & 15.4484 & 15.4303 & 15.4256 & 15.4244 & 15.4257 \\
\hline 1SE & 0.0002 & 0.0002 & 0.0002 & 0.0001 & 0.0001 & 0.0002 & 0.0002 & 0.0001 & 0.0002 & 0.0002 & 0.0002 & 0.0002 \\
\hline${ }^{208} \mathrm{~Pb} /{ }^{204} \mathrm{~Pb}$ & 37.0810 & 37.0997 & 37.0713 & 37.0718 & 37.1067 & 37.0846 & 37.0885 & 37.1063 & 36.7585 & 36.7132 & 36.7114 & 36.7181 \\
\hline 1SE & 0.0005 & 0.0004 & 0.0005 & 0.0003 & 0.0003 & 0.0004 & 0.0006 & 0.0003 & 0.0004 & 0.0004 & 0.0004 & 0.0004 \\
\hline
\end{tabular}

Note: SE stands for standard error; $\quad \varepsilon N d=\left[\left({ }^{143} \mathrm{Nd} /{ }^{144} \mathrm{Nd}\right)_{\text {Sample }} /\left({ }^{143} \mathrm{Nd} /{ }^{144} \mathrm{Nd}\right)_{\mathrm{CHUR}}-1\right] \times 10^{4} ; \quad\left({ }^{443} \mathrm{Nd} / /^{144} \mathrm{Nd}\right)_{\mathrm{CHUR}}=0.512638$;

$\varepsilon \mathrm{Hf}=\left[\left({ }^{176} \mathrm{Hf} /{ }^{177} \mathrm{Hf}\right)_{\text {Sample }} /\left({ }^{176} \mathrm{Hf} /{ }^{177} \mathrm{Hf}\right)_{\mathrm{CHUR}}-1\right] \times 10^{4} ; \quad\left({ }^{4} ; \mathrm{Hf} /{ }^{177} \mathrm{Hf}\right)_{\mathrm{CHUR}}=0.282772$. 
Table DR4 Geochemical characteristics of some high-Mgandesites (wt.\%) from subduction zone

\begin{tabular}{c|c|c|c|c|c|c|c}
\hline Location & $\mathrm{SiO}_{2}$ & $\mathrm{Al}_{2} \mathrm{O}_{3}$ & $\mathbf{M g O}$ & $\mathrm{CaO}$ & $\mathrm{Na} 2 \mathrm{O}+\mathrm{K}_{2} \mathrm{O}$ & $\mathbf{M g} \#$ & $\mathrm{La} / \mathrm{Yb}$ \\
\hline White Island,New Zealand & 55.6 & 13.2 & 10.1 & 8.9 & 3.5 & 0.71 & 3.6 \\
\hline Amphlett Island,Papua New Guinea & 54.3 & 14.8 & 8.7 & 7.6 & 5.2 & 0.67 & - \\
\hline Mount Shasta,Cascades & 57.9 & 14.7 & 8.9 & 8.1 & 3.9 & 0.74 & 10.2 \\
\hline Setouchi Belt,Japan & 58.5 & 13.3 & 9.5 & 6.1 & 3.9 & 0.73 & 9.1 \\
\hline Adak Island,central Aleutians & 56.8 & 15.5 & 7.4 & 8.6 & 4.4 & 0.66 & 8.8 \\
\hline Piipvolcano,western Aleutians & 57.0 & 16.0 & 6.6 & 7.7 & 4.8 & 0.72 & 4.6 \\
\hline Russian Far East & $\mathbf{5 5 . 4 - 5 7 . 3}$ & $\mathbf{1 4 . 6 - 1 5 . 0}$ & $\mathbf{4 . 9 - 5 . 8}$ & $\mathbf{5 . 8 - 6 . 6}$ & $\mathbf{5 . 2 - 6 . 4}$ & $\mathbf{0 . 5 3 - 0 . 5 6}$ & $\mathbf{1 8 - 2 3}$ \\
\hline
\end{tabular}

Data sources: Wood and Tuner (2009). 
Table DR5 Compared Sr-Nd-Pb isotopic data from intraplate basalts in NE China and andesites in arc

\begin{tabular}{|c|c|c|c|c|c|c|c|c|}
\hline Location & Lithology & Samp1e & $\left({ }^{87} \mathrm{Sr} l^{86} \mathrm{Sr}\right) \mathrm{i}$ & $\left({ }^{143} \mathrm{Nd} /{ }^{144} \mathrm{Nd}\right) \mathrm{i} /[\delta \mathrm{Nd}(\mathrm{t})]$ & ${ }^{206} \mathrm{~Pb} /{ }^{204} \mathrm{~Pb}$ & ${ }^{207} \mathrm{~Pb} /{ }^{204} \mathrm{~Pb}$ & ${ }^{208} \mathrm{~Pb} /{ }^{204} \mathrm{~Pb}$ & Reference \\
\hline \multirow{13}{*}{ Changbaishan } & \multirow{13}{*}{ sodic basalts } & HaKu-1a & 0.705078 & 0.512537 & 17.379 & 15.538 & 37.743 & \multirow{13}{*}{ Kuritani et al.,2009 } \\
\hline & & Haku-2 & 0.705181 & 0.512513 & 17.482 & 15.543 & 37.805 & \\
\hline & & Haku-7 & 0.704956 & 0.512582 & 17.410 & 15.540 & 37.893 & \\
\hline & & Haku-10 & 0.705008 & 0.512599 & 17.384 & 15.506 & 37.781 & \\
\hline & & Haku-20 & 0.705319 & 0.512550 & 17.569 & 15.508 & 37.953 & \\
\hline & & HaKu-25 & 0.704985 & 0.512555 & 17.390 & 15.535 & 37.627 & \\
\hline & & Haku-26 & 0.705039 & 0.512555 & 17.378 & 15.538 & 37.625 & \\
\hline & & Haku-27 & 0.704926 & 0.512642 & 17.824 & 15.537 & 38.244 & \\
\hline & & Haku-30b & 0.705018 & 0.512579 & 17.435 & 15.532 & 37.672 & \\
\hline & & Haku-32b & 0.705531 & 0.512554 & 17.614 & 15.541 & 38.087 & \\
\hline & & Haku-34 & 0.705114 & 0.512583 & 17.657 & 15.543 & 38.008 & \\
\hline & & Haku-35 & 0.704817 & 0.512586 & 17.373 & 15.526 & 37.564 & \\
\hline & & Haku-36 & 0.704954 & 0.512619 & 17.604 & 15.545 & 37.845 & \\
\hline \multirow{4}{*}{ Longgang } & \multirow{4}{*}{ sodic basalts } & LG9940 & 0.704467 & 0.512665 & 17.9100 & 15.5700 & 38.3940 & \multirow{4}{*}{ Kuritani et al.,2011 } \\
\hline & & LG99L11-1 & 0.704443 & 0.512674 & 17.9530 & 15.5560 & 38.4800 & \\
\hline & & HJ97001A & 0.704488 & 0.512663 & 17.9310 & 15.5460 & 38.4570 & \\
\hline & & HAKU40 & 0.704418 & 0.512723 & 18.1417 & 15.5566 & 38.5694 & \\
\hline \multirow{11}{*}{ Shuangliao } & \multirow{11}{*}{ sodic basalts } & BBT-3 & 0.703298 & $0.512838 \quad(5.2)$ & 18.24 & 15.47 & 38.21 & \multirow{11}{*}{ Xu et al., 2012} \\
\hline & & BLS-5 & 0.70341 & $0.512892 \quad(6.2)$ & 18.13 & 15.50 & 38.18 & \\
\hline & & DHLB-1 & 0.703526 & 0.512831 & 18.34 & 15.50 & 38.33 & \\
\hline & & DHLB-8 & 0.703597 & 0.512854 (5.5) & 18.28 & 15.50 & 38.34 & \\
\hline & & DTJ-5 & 0.703609 & 0.512821 (4.6) & 18.26 & 15.48 & 38.23 & \\
\hline & & NBS-3 & 0.703043 & $0.512845 \quad(5.3)$ & & & & \\
\hline & & NBS-4 & 0.703254 & $0.512854 \quad(5.4)$ & 18.23 & 15.46 & 38.19 & \\
\hline & & XHLB-1 & 0.703407 & 0.512811 (4.7) & 18.39 & 15.56 & 38.58 & \\
\hline & & XHLB-7 & 0.703353 & $0.51282 \quad(4.8)$ & 18.29 & 15.49 & 38.29 & \\
\hline & & XTJ-1 & 0.703278 & $0.512798 \quad(4.2)$ & 18.21 & 15.48 & 38.21 & \\
\hline & & XTJ-6 & 0.703311 & $0.512829 \quad(4.8)$ & 18.21 & 15.49 & 38.25 & \\
\hline \multirow{6}{*}{$\begin{array}{l}\text { Mt. Shasta, } \\
\text { N California }\end{array}$} & \multirow{6}{*}{ andesites } & $85-48 b$ & 0.70275 & 7.4 & 18.798 & 15.589 & 38.407 & \multirow{6}{*}{ Grove et al., 2002} \\
\hline & & $82-94 a$ & 0.70366 & 5.2 & 18.937 & 15.616 & 38.573 & \\
\hline & & $82-94 d$ & 0.70292 & 7.2 & 18.893 & 15.611 & 38.56 & \\
\hline & & $85-41 b$ & 0.70299 & 6.6 & 18.851 & 15.567 & 38.405 & \\
\hline & & $82-85$ & 0.70375 & 4.5 & 18.96 & 15.595 & 38.579 & \\
\hline & & $85-59$ & 0.70378 & 5.1 & 18.991 & 15.634 & 38.707 & \\
\hline \multirow{7}{*}{$\begin{array}{l}\text { Western } \\
\text { Aleutian }\end{array}$} & & Apr-16 & 0.7028 & 10.29 & 17.987 & 15.412 & 37.448 & \multirow{7}{*}{ Yogodzinski et al.,1994 } \\
\hline & & V35/G5B & 0.70264 & 10.19 & 18.089 & 15.431 & 37.535 & \\
\hline & & V35/G5A & 0.70265 & 10.64 & 18.081 & 15.43 & 37.539 & \\
\hline & andesites & V35/G4X1 & 0.70269 & 10.64 & 18.063 & 15.422 & 37.487 & \\
\hline & & V35/G9D & 0.70257 & 10.48 & 18.165 & 15.422 & 37.56 & \\
\hline & & V35/G7C & 0.70269 & 10.44 & 18.161 & 15.438 & 37.594 & \\
\hline & & V35/G7A & 0.70276 & 11.15 & 18.015 & 15.42 & 37.449 & \\
\hline
\end{tabular}


Table DR6 Present-day compositions of the end-member components

\begin{tabular}{cccc}
\hline Component & 1.5 Ga pelagic sediments & Recent Pacific sediments & Depleted mantle \\
\hline $\mathrm{Sr}, \mathrm{ppm}$ & 189 & 189 & 7.66 \\
$\mathrm{Nd}, \mathrm{ppm}$ & 46 & 46 & 0.58 \\
$\mathrm{~Pb}, \mathrm{ppm}$ & 18 & 18 & 0.02 \\
& & & \\
${ }^{87} \mathrm{Sr} /{ }^{86} \mathrm{Sr}$ & 0.72030 & 0.70954 & 0.70369 \\
${ }^{143} \mathrm{Nd} /{ }^{144} \mathrm{Nd}$ & 0.51170 & 0.51234 & 0.51285 \\
${ }^{206} \mathrm{~Pb} /{ }^{204} \mathrm{~Pb}$ & 16.757 & 18.827 & 17.645 \\
${ }^{207} \mathrm{~Pb} /{ }^{204} \mathrm{~Pb}$ & 15.478 & 15.672 & 15.398 \\
${ }^{208} \mathrm{~Pb} /{ }^{204} \mathrm{~Pb}$ & 37.239 & 39.395 & 37.360 \\
\hline
\end{tabular}

Data from Kuritani et al. (2011). 